\title{
Terapia de relaxamento muscular de Jacobson em pessoas com ansiedade: revisão integrativa
}

\author{
Jacobson's muscle relaxation therapy in people with anxiety: an integrative review
}
Terapia de relajación muscular de Jacobson en personas con ansiedad: una revisión integradora

Bruno Rodrigues Bezerra ${ }^{1 *}$, Aline Raquel de Sousa lbiapina ${ }^{1}$, Ana Paula Cardoso Costa ${ }^{1}$, José de Siqueira Amorim Júnior ${ }^{1}$, Rodrigo Aragão da Silva ${ }^{1}$, Lany Leide de Castro Rocha Campelo ${ }^{1}$, Antonio Alberto Ibiapina Costa Filho ${ }^{1}$, Larissa Vanessa Ferreira Memoria ${ }^{1}$

\begin{abstract}
RESUMO
Objetivo: Analisar os efeitos da terapia de relaxamento muscular de Jacobson em indivíduos ansiosos. Métodos: Revisão integrativa guiada pelo protocolo Preferred Reporting Items for Systematic Reviews and Meta-Analysis. A busca foi realizada nas bases de dados: Medical Literature Analysis and Retrievel System Online (PubMed)/MEDLINE), Literatura Latino-americana e do Caribe em Ciências da Saúde (LILACS) e na Biblioteca Virtual em Saúde (BVS), no período de agosto e setembro de 2019. A avaliação da qualidade dos estudos foi avaliada pelo nível de evidência conforme o Oxford Centre for Evidence - based medicine. A análise crítica das informações extraídas e a síntese qualitativa dos estudos selecionados foram realizadas de forma descritiva. Resultados: treze estudos atenderam aos critérios de inclusão. De modo geral, os resultados revelaram um efeito positivo da técnica de relaxamento muscular progressivo de Jacobson na redução dos sintomas de ansiedade na população. Os indivíduos com diversas patologias crônicas que receberam relaxamento muscular apresentaram redução dos sintomas ansiosos. Considerações finais: $O$ relaxamento muscular progressivo de Jacobson é notado como um método eficaz para auxiliar na redução dos sintomas de ansiedade, bem como na melhoria da qualidade de vida e recuperação da saúde do usuário.
\end{abstract}

Palavras-chave: Ansiedade, Saúde mental, Terapia de relaxamento.

\begin{abstract}
Objective: To analyze the effects of Jacobson's muscle relaxation therapy on anxious individuals. Methods: Integrative review guided by the Preferred Reporting Items for Systematic Reviews and Meta-Analysis protocol. The search was carried out in the databases: Medical Literature Analysis and Retrievel System Online (PubMed) / MEDLINE), Latin American and Caribbean Literature in Health Sciences (LILACS) and in the Virtual Health Library (VHL), in the period of August and September 2019. The evaluation of the quality of the studies was assessed by the level of evidence according to the Oxford Center for Evidence-based Medicine. The critical analysis of the extracted information and the qualitative synthesis of the selected studies were carried out descriptively. Results: thirteen studies met the inclusion criteria. In general, the results revealed a positive effect of Jacobson's progressive muscle relaxation technique in reducing anxiety symptoms in the population. Individuals with several chronic pathologies who received muscle relaxation had reduced anxiety symptoms. Final considerations: Jacobson's progressive muscle relaxation is noted as an effective method to assist in reducing anxiety symptoms, as well as improving the quality of life and recovering the health of the user.
\end{abstract}

Keywords: Anxiety, Mental health, Relaxation therapy.

${ }^{1}$ Universidade Federal do Piauí (UFPI), Picos - Piauí.

*E-mail: brunnobezerra@outlook.com

SUBMETIDO EM: $3 / 2020$

ACEITO EM: 4/2020

PUBLICADO EM: 6/2020 


\section{RESUMEN}

Objetivo: analizar los efectos de la terapia de relajación muscular de Jacobson en individuos ansiosos. Métodos: Revisión integradora guiada por los elementos de informes preferidos para revisiones sistemáticas y el protocolo de metanálisis. La búsqueda se realizó en las bases de datos: Análisis de literatura médica y sistema de recuperación en línea (PubMed) / MEDLINE), Literatura latinoamericana y caribeña en ciencias de la salud (LILACS) y en la Biblioteca virtual de salud (BVS), en el período de Agosto y septiembre de 2019. La evaluación de la calidad de los estudios se evaluó según el nivel de evidencia según el Centro de Oxford para Medicina Basada en la Evidencia. El análisis crítico de la información extraída y la síntesis cualitativa de los estudios seleccionados se realizaron de forma descriptiva. Resultados: trece estudios cumplieron los criterios de inclusión. En general, los resultados revelaron un efecto positivo de la técnica de relajación muscular progresiva de Jacobson en la reducción de los síntomas de ansiedad en la población. Las personas con varias patologías crónicas que recibieron relajación muscular habían reducido los síntomas de ansiedad. Consideraciones finales: la relajación muscular progresiva de Jacobson se observa como un método eficaz para ayudar a reducir los síntomas de ansiedad, así como a mejorar la calidad de vida y recuperar la salud del usuario.

Palabras clave: Ansiedad, Salud mental, Terapia de relajación.

\section{INTRODUÇÃO}

Com o advento da Reforma Psiquiátrica Brasileira em meados dos anos setenta, inúmeros avanços surgiram na atenção voltada aos indivíduos com transtornos mentais. Até então, 0 tratamento hospitalocêntrico, privavam os pacientes de exercerem seus direitos. No entanto, com a Política Nacional de Saúde Mental (PNSM) foi possível modificar essa realidade e promover significantes mudanças nesse cenário, onde os usuários passaram a receber amparo e serem reinseridos no ambiente familiar e comunitário (BRASIL, 2011). Por meio da Política Nacional de Saúde Mental, o Ministério da Saúde (MS), mediante a Portaria no 3.088/2011, instituiu a Rede de Atenção Psicossocial (RAPS) com a finalidade de criar, ampliar e articular pontos de atenção à saúde para pessoas com sofrimento ou transtorno mental e com necessidades decorrentes do uso de drogas no âmbito do Sistema Único de Saúde (SUS) (BRASIL, 2011).

A RAPS oferece uma variedade de cuidados aos pacientes e familiares. A rede de atendimento compõese dos seguintes serviços de atenção: Centros de Atenção Psicossocial (CAPS); Serviços Residenciais Terapêuticos (SRT); Centros de Convivência e Cultura; Atenção de Urgência e Emergência; Atenção Hospitalar e Atenção Básica (MOREIRA MIB; CAMPOS T, 2017). Dados epidemiológicos da Organização Mundial de Saúde (OMS), em 2015, apontaram que cerca de 254 milhões de pessoas em todo o mundo apresentavam algum transtorno de ansiedade. No Brasil, estima-se que 9,3\% da população é acometida por este transtorno (WHO, 2017).

A ansiedade é um transtorno mental comum definida como um sistema complexo de respostas cognitivas, afetivas, fisiológicas e comportamentais, podendo ser ocasionada em decorrência de alguma circunstância vivenciada anteriormente a qual ocasionou uma aversão, ameaçando dessa forma os interesses vitais da pessoa. Além disso, a ansiedade pode tornar-se patológica e ocasionar consequências no âmbito social e ocupacional do indivíduo (MULLER JL, et al., 2015). Os sintomas de ansiedade podem variar em estágios leve, moderado e grave com duração de meses a anos. Caracterizam-se por manifestações de emoções frequentes diante de situações desencadeantes ou precipitantes, ocasionando sinais de palpitações, taquicardia, palidez, dispneia, tensão muscular, tremor, desordens intestinais, dentre outros (WHO, 2017).

Segundo Caixeta PPP (2014), os sintomas de ansiedade são comuns entre a população. Diante disso, a detecção precoce de pessoas que apresentam a sintomatologia é de suma importância, pois possibilita que os profissionais de saúde, dentre eles o enfermeiro, direcione-os para um tratamento adequado. Dentre as terapias complementares disponíveis para tratar a ansiedade, as técnicas de relaxamento muscular progressivo apresentam-se como importante método terapêutico. Através dessas técnicas o indivíduo pode aprender e executá-las ao longo do dia, sem necessitar da presença de um profissional de saúde, pois é um método não invasivo, que auxilia no equilíbrio mental e corporal, diminuindo a angústia e a emotividade. $O$ enfermeiro exerce um papel estratégico e indispensável para a saúde, através do exercício de práticas assistenciais, educativas e preventivas (BARBIANI R; NORA CRD; SCHAEFER R, 2016) e utilização de métodos não farmacológicas para resolução de diagnósticos identificados ou para manutenção e recuperação da saúde, como é o caso da técnica de relaxamento muscular, visando o tratamento da ansiedade (GOMES MIS; CRUZ ICF, 2015). 
O uso da técnica de relaxamento muscular de Jacobson, também chamada de Relaxamento Progressivo, consiste numa série de procedimentos que desencadeiam a redução da tensão muscular em grupos musculares específicos. De forma mais detalhada, pode-se inferir que a partir de um treinamento guiado, a técnica possibilita ao sujeito identificar as regiões tensas do seu corpo e direcionar exercícios voltados para a descontração muscular e o alívio da tensão (PIFARRÉ P, et al., 2015). A Técnica de Relaxamento Muscular de Jacobson é simples de fazer e ensinar. Consiste em no mínimo de 6 a 12 sessões. O paciente é solicitado a tensionar cada grupo muscular específico por 5 a 10 segundos e depois relaxar por 20 a 30 segundos. Recomenda-se ainda que essa técnica seja realizada pelo menos 2 vezes na semana (KESTENBERG CCF, et al., 2014).

Como resultados da aplicação dessa técnica, observou-se a diminuição do ritmo cardíaco e um aumento da percepção de relaxamento muscular global e nos grupos musculares trabalhados (BORGES EMN, FERREIRA TJR, 2013), bem como uma sensação de relaxamento físico e mental, demonstrando ser eficaz no auxílio do tratamento de indivíduos com sintomas de ansiedade, tensão muscular, além de outras alterações (VASCONCELOS LHS, 2014). Dessa forma, o objetivo deste estudo consiste em analisar as evidências disponíveis na literatura sobre a realização da terapia de relaxamento muscular em indivíduos ansiosos.

\section{MÉTODOS}

O estudo caracteriza-se como uma revisão integrativa, foi operacionalizado por meio de seis etapas as quais estão estreitamente interligadas: elaboração da pergunta norteadora, busca na literatura, coleta de dados, análise crítica dos estudos incluídos, discussão dos resultados e apresentação da revisão integrativa (SOUZA MT; SILVA MP; CARVALHO R, 2010).Para a elaboração da pergunta de pesquisa, utilizou-se a estratégia PICO, um acrônimo que contém os elementos fundamentais para a busca de evidências: PPACIENTE; I- INTERVENÇÃO; C-COMPARAÇÃO; O-OUTCOME. No presente estudo, a estratégia se apresenta: P- Indivíduos com sintomas ansiosos; I- Terapia de Relaxamento Muscular; C- Não houve comparação e O- Redução dos sintomas. O desfecho primário focou na redução dos sintomas de ansiedade em adultos submetidos à terapia de relaxamento muscular.

A partir disso foi elaborada a seguinte questão de estudo: Como a técnica de relaxamento muscular é utilizada para reduzir sintomas ansiosos? A coleta de dados ocorreu entre o mês de agosto e setembro de 2019. As bases de dados utilizadas foram: PubMed/MEDLINE, LILACS. A BVS também foi utilizada para selecionar estudos utilizados na presente revisão.Os seguintes descritores controlados foram utilizados: Ansiedade, Saúde Mental, Terapia de Relaxamento; Anxiety, Mental Health, Relaxation Therapy. A sintaxe das buscas em cada base de dados encontra-se descrita no (Quadro 1).

Quadro 1 - Sintaxes empregadas nas respectivas bases de dados durante a busca de estudos. Picos, PI, Brasil, 2019.

\begin{tabular}{|c|l|}
\hline Base de dados & \multicolumn{1}{|c|}{ Sintaxe de Pesquisa } \\
\hline & (("anxiety"[MeSH Terms] OR "anxiety"[All Fields]) AND ("mental health"[MeSH \\
Terms] OR ("mental"[All Fields] AND "health"[All Fields]) OR "mental health"[All \\
& $\begin{array}{c}\text { Fields])) AND ("relaxation therapy"[MeSH Terms] OR ("relaxation"[All Fields] AND } \\
\text { "therapy"[All Fields]) OR "relaxation therapy"[All Fields]). }\end{array}$ \\
\hline LILACS & (tw:(anxiety)) AND (tw:(mental health)) AND (tw:(relaxation therapy)) \\
\hline BVS & (tw:(ansiedade)) AND (tw:(saúde mental)) AND (tw:(terapia de relaxamento)) \\
\hline
\end{tabular}

Fonte: Bezerra BR, et al., 2020.

Os critérios de inclusão utilizados na seleção foram: estudos primários, disponíveis em sua totalidade, publicados até setembro de 2019 em qualquer idioma. Foram excluídos da busca inicial capítulos de livros, teses de doutorado, dissertações de mestrado e relatórios técnicos. As pesquisas ocorreram por dois pesquisadores simultaneamente. A estratégia de busca foi conduzida por meio de recomendações propostas pelo protocolo Preferred Reporting Items for Systematic Reviews and Meta-Analyses (PRISMA) (MOHER D et al., 2009). 
A análise para seleção dos estudos foi realizada em três fases, a saber:

1. Os estudos selecionados nas bases de dados e na BVS foram analisados e pré-selecionados segundo os critérios de inclusão e exclusão, por meio da leitura de seus títulos e resumos. Caso o artigo não possuísse resumo ou este não permitisse a exclusão ou inclusão dele, realizava-se a leitura do artigo completo. Obtevese 346 estudos na PubMed, 100 na LILACS e 100 na BVS, totalizando 546 estudos.

2. Na segunda fase os estudos foram analisados quanto ao potencial de participação no estudo, avaliando o atendimento a questão de pesquisa, bem como o tipo de investigação, objetivos, amostra, método, desfechos, resultados e conclusão, resultando em 25 artigos.

3. A terceira fase consistiu na leitura integral dos 25 textos, visando a coleta de dados específicos aos objetivos da revisão, chegando aos 13 estudos selecionados para a pesquisa.

Para coleta de informações dos estudos selecionados, os pesquisadores elaboraram e utilizaram um instrumento contendo as seguintes informações: autores, ano de publicação, periódico, país, tipo de estudo, principais resultados e nível de evidência.

Nesse estudo tomou como base o nível de evidência determinado conforme o que é estabelecido por Oxford Centre for Evidence-based Medicine (2009), de modo que: 1A - revisão sistemática de ensaios clínicos controlados randomizados; 1B - ensaio clínico controlado randomizado com intervalo de confiança estreito; $1 \mathrm{C}$ - resultados terapêuticos do tipo "tudo ou nada"; $2 \mathrm{~A}$ - revisão sistemática de estudos de coorte; $2 \mathrm{~B}$ estudo de coorte (incluindo ensaio clínico randomizado de menor qualidade); $2 \mathrm{C}$ - observação de resultados terapêuticos ou estudos ecológicos; 3A - revisão sistemática de estudos caso-controle; 3B - estudo casocontrole; 4 - relato de casos (incluindo coorte ou caso-controle de menor qualidade); 5 - opinião de especialistas. Com a intenção de diminuir prováveis erros sistemáticos na interpretação dos resultados e no delineamento dos estudos, a pesquisa foi realizada por dois revisores de forma independente, de modo a garantir o rigor do método e a fidedignidade dos resultados.

Os artigos foram selecionados por meio da sequência: leitura de título, resumo e leitura do texto integral. Nos casos em que ocorreram desacordos, houve discussão entre os avaliadores para alcançar um consenso. A análise crítica das informações extraídas e a síntese qualitativa dos estudos selecionados foram realizadas de forma descritiva. As estratégias de inclusão e exclusão utilizadas nas bases de dados encontram-se apresentadas no fluxograma em destaque na (Figura 1), conforme recomendações do grupo PRISMA. 
Figura 1 - Fluxograma do processo de seleção dos estudos, segundo o PRISMA. Picos, PI, Brasil, 2020.

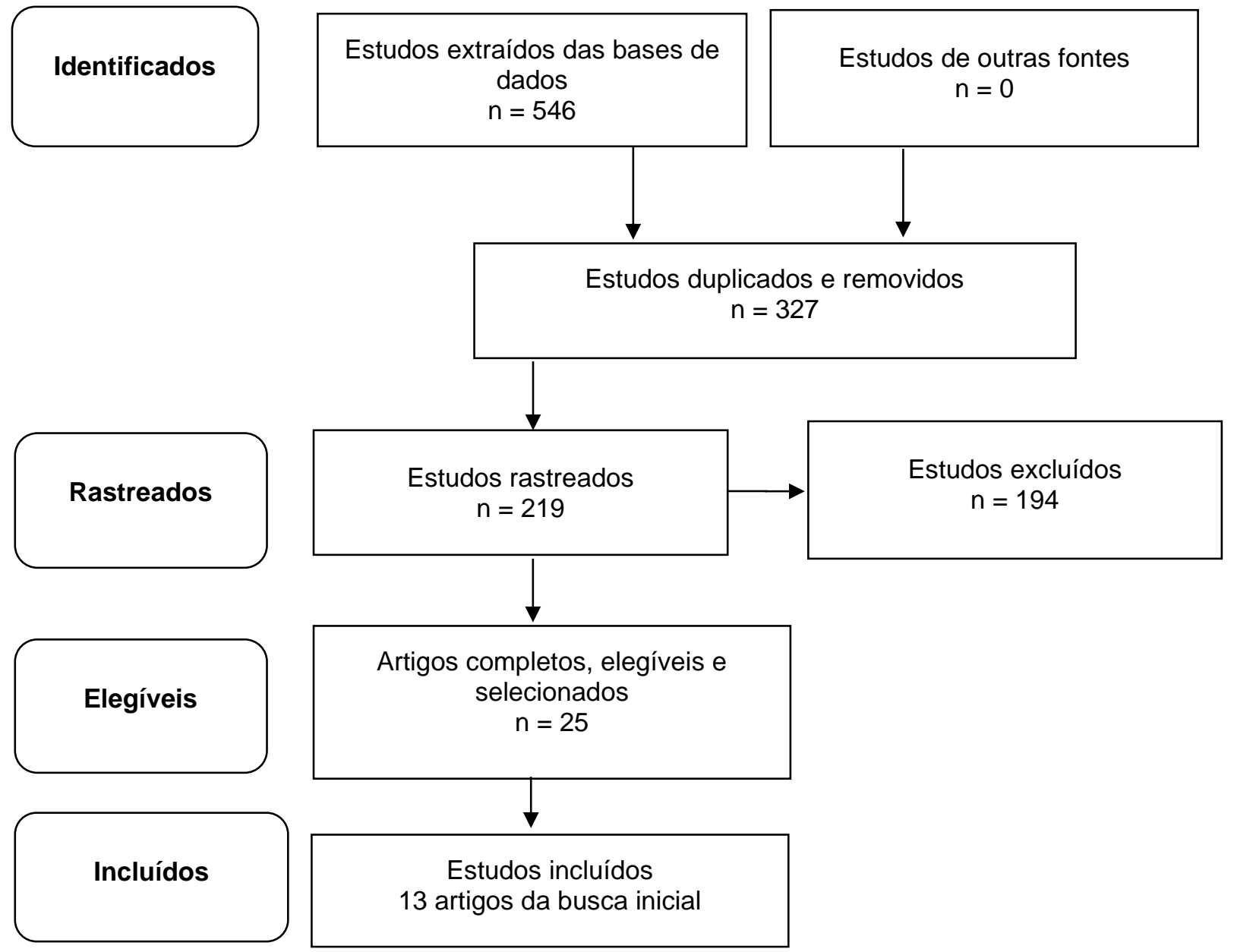

Fonte: Bezerra BR, et al., 2020.

\section{RESULTADOS E DISCUSSÃO}

Nos achados pode-se identificar que a maioria dos estudos foi publicada em 2019 , sendo $18,52 \%$ ( $n=5$ ), observou-se, também, que $37,4 \%(n=10)$ foram publicados em periódicos internacionais. Quanto ao delineamento, $14,81 \%$ são estudos randomizados $(n=4)$. Sobre o nível de evidência, observou-se que $14,81 \%$ $(n=4)$ dos artigos analisados apresentou avaliação 1B e 2C.

A seguir, o Quadro 2 apresenta os estudos segundo os autores, ano, periódico, país, tipo de estudo, principais resultados e nível de evidência. 
Quadro 2 - Quadro-síntese das características dos estudos incluídos na revisão - Picos, PI, Brasil, 2020.

\begin{tabular}{|c|c|c|c|c|c|}
\hline $\begin{array}{l}\text { Autor } \\
\text { (ano) }\end{array}$ & Periódico & País & Tipo de estudo & Principais resultados & $\begin{array}{c}\text { Nível de } \\
\text { evidência }\end{array}$ \\
\hline $\begin{array}{l}\text { Pouraboli B, Poodineh Z, } \\
\text { Jahani Y (2019) }\end{array}$ & $\begin{array}{l}\text { Asian Pac J } \\
\text { Cancer Prev }\end{array}$ & Irã & $\begin{array}{l}\text { Randomizado } \\
\text { controlado }\end{array}$ & $\begin{array}{l}\text { Houve uma diferença estatisticamente significante entre a } \\
\text { característica de ansiedade, fadiga e qualidade do sono nos grupos } \\
\text { intervenção e controle após a intervenção }(p>0,05) \text {. Nesse sentido, a } \\
\text { técnica de relaxamento muscular aplicada aos pais de crianças com } \\
\text { câncer melhorou o funcionamento da família e a saúde mental. }\end{array}$ & $1 \mathrm{~B}$ \\
\hline Hampson N, et al. (2019) & $\begin{array}{l}\text { Disabil } \\
\text { Rehabil }\end{array}$ & $\begin{array}{l}\text { Reino } \\
\text { Unido }\end{array}$ & $\begin{array}{l}\text { Randomizado } \\
\text { controlado }\end{array}$ & $\begin{array}{l}\text { Os resultados apresentaram melhorias estatisticamente significativas } \\
\text { nas medidas de ansiedade e depressão após a conclusão das sessões } \\
\text { de relaxamento em pessoas que vivem com condições neurológicas a } \\
\text { longo prazo. }\end{array}$ & $1 \mathrm{~B}$ \\
\hline Merakou K, et al. (2019) & Explore (NY) & Grécia & $\begin{array}{l}\text { Estudo de } \\
\text { intervenção }\end{array}$ & $\begin{array}{l}\text { O grupo intervenção apresentou uma diminuição nos sintomas de } \\
\text { depressão, ansiedade e estresse }(p<0,001) \text {. Nenhuma mudança } \\
\text { significativa foi observada no grupo controle durante o período de } \\
\text { acompanhamento em pessoas desempregadas de longa duração com } \\
\text { transtornos de ansiedade. }\end{array}$ & $2 \mathrm{C}$ \\
\hline Zhao L, et al. (2012) & $\begin{array}{l}\text { Eur J Obstet } \\
\text { Gynecol } \\
\text { Reprod Biol }\end{array}$ & China & $\begin{array}{l}\text { Randomizado } \\
\text { controlado }\end{array}$ & $\begin{array}{l}\text { Aplicação do treinamento de relaxamento muscular progressivo (PMR) } \\
\text { na ansiedade, depressão e qualidade de vida (QV) de pacientes com } \\
\text { endometriose sob terapia agonista do hormônio liberador de } \\
\text { gonadotrofina }(\mathrm{GnRH}) \text {. Após } 12 \text { semanas de intervenção, ambos os } \\
\text { grupos apresentaram melhora significativa na ansiedade, depressão e } \\
\mathrm{QV}(\mathrm{P}<0,05) \text {, mostrando que essa técnica foi eficaz. }\end{array}$ & $1 \mathrm{~B}$ \\
\hline $\begin{array}{l}\text { Borges EMN e Ferreira TJR } \\
\qquad(2013)\end{array}$ & $\begin{array}{l}\text { Revista } \\
\text { Portuguesa } \\
\quad \text { de } \\
\text { Enfermagem } \\
\text { de Saúde } \\
\text { Mental }\end{array}$ & Portugal & Longitudinal & $\begin{array}{l}\text { O uso da técnica de relaxamento muscular de Jacobson associada a } \\
\text { técnica de imaginação guiada em um grupo de enfermeiros e } \\
\text { estudantes de enfermagem resultou na diminuição do ritmo cardíaco } \\
\text { e em maior percepção de relaxamento muscular nos diferentes } \\
\text { grupos musculares trabalhados pelos participantes. }\end{array}$ & $2 \mathrm{C}$ \\
\hline Félix MMS, et al. (2018) & $\begin{array}{l}\text { Revista } \\
\text { Latino- } \\
\text { Americana de } \\
\text { Enfermagem }\end{array}$ & Brasil & $\begin{array}{l}\text { Ensaio clínico } \\
\text { randomizado }\end{array}$ & $\begin{array}{l}\text { A terapia de relaxamento muscular associada a terapia de relaxamento } \\
\text { com imagem guiada mostrou evidências importantes quanto a redução } \\
\text { dos níveis de ansiedade e cortisol pré-operatórios em pessoas obesas } \\
\text { submetidas à cirurgia bariátrica. }\end{array}$ & $1 \mathrm{~B}$ \\
\hline
\end{tabular}

REAS/EJCH | Vol.Sup.n.51 | e3438 | DOI: https://doi.org/10.25248/reas.e3438.2020 Página 6 de 11 


\begin{tabular}{|c|c|c|c|c|c|}
\hline Freitas GR, et al. (2018) & $\begin{array}{l}\text { Psicologia } \\
\text { escolar e } \\
\text { educacional }\end{array}$ & Brasil & $\begin{array}{l}\text { Estudo de } \\
\text { coorte }\end{array}$ & $\begin{array}{l}\text { A técnica de relaxamento muscular progressivo contribuiu para a } \\
\text { redução da ansiedade e estresse em professores de escolas públicas } \\
\text { em exercício de suas funções. }\end{array}$ & $2 \mathrm{~B}$ \\
\hline $\begin{array}{c}\text { Gomes MIS; Cruz ICF } \\
\text { (2015) }\end{array}$ & $\begin{array}{l}\text { Boletim } \\
\text { Nepae- } \\
\text { Nessen }\end{array}$ & Brasil & Descritivo & $\begin{array}{l}\text { A aplicação da técnica de relaxamento muscular em clientes no pré- } \\
\text { operatório de cirurgia cardíaca mostrou-se eficaz quanto ao } \\
\text { relaxamento da mente e do corpo dos clientes. }\end{array}$ & 4 \\
\hline $\begin{array}{l}\text { Norelli SK, e Krepps JM. } \\
\text { (2019) }\end{array}$ & Stat Pearls & $\begin{array}{l}\text { Estados } \\
\text { Unidos }\end{array}$ & Descritivo & $\begin{array}{l}\text { Os autores descreveram três técnicas de relaxamento para ajudar os } \\
\text { pacientes com sintomas como ansiedade, depressão, estresse, entre } \\
\text { outros. Dentre essas a de relaxamento muscular de Jacobson pode ser } \\
\text { utilizada para minimizar essa sintomatologia. }\end{array}$ & 4 \\
\hline Parás-Bravo P. et al. (2017) & PLos One & Espanha & $\begin{array}{l}\text { Estudo de } \\
\text { intervenção } \\
\text { multicêntrico }\end{array}$ & $\begin{array}{l}\text { Os autores evidenciaram a eficácia da técnica de relaxamento } \\
\text { muscular na melhoria da qualidade de vida em uma amostra de } \\
\text { pacientes oncológicos com sintomas ansiosos e depressivos tratados } \\
\text { no Sistema Nacional de Saúde Pública da Espanha. }\end{array}$ & 20 \\
\hline Parás-bravo P. et al. (2018) & $\begin{array}{l}\text { BMC } \\
\text { Complement } \\
\text { Altern. Med. }\end{array}$ & Espanha & $\begin{array}{l}\text { Estudo de } \\
\text { intervenção } \\
\text { multicêntrico }\end{array}$ & $\begin{array}{l}\text { Evidenciou que a técnica de relaxamento muscular de Jacobson pode } \\
\text { auxiliar a diminuir o consumo de drogas psicotrópicas e analgésicas } \\
\text { em pacientes com câncer e com sintomas de ansiedade. }\end{array}$ & 20 \\
\hline Pifarré P. et al. (2015) & $\begin{array}{c}\text { Eur. } \\
\text { Psiquiatria }\end{array}$ & Espanha & $\begin{array}{l}\text { Estudo de } \\
\text { coorte }\end{array}$ & $\begin{array}{l}\text { Através dos testes realizados, os autores evidenciaram que o } \\
\text { relaxamento induzido por um procedimento físico/psicológico pode ser } \\
\text { tão eficaz quanto a administração de um ansiolítico de referência na } \\
\text { redução da atividade cerebral durante um estado estressante e } \\
\text { ansioso, em usuários com câncer. }\end{array}$ & $2 \mathrm{~B}$ \\
\hline Wilczyńska D. et al. (2019) & $\begin{array}{l}\text { International } \\
\text { Journal of } \\
\text { Occupational } \\
\text { Medicine and } \\
\text { Environmenta } \\
\text { I Health }\end{array}$ & Polônia & $\begin{array}{l}\text { Estudo de } \\
\text { coorte }\end{array}$ & $\begin{array}{l}\text { Os autores realizaram um comparativo entre a utilização da técnica de } \\
\text { relaxamento muscular pós-isométrico e a técnica de relaxamento } \\
\text { muscular de Jacobson, chegando à conclusão de que ambas foram } \\
\text { métodos eficazes para minimizar os efeitos negativos do estresse } \\
\text { mediados pela ansiedade, nos pacientes com tensão psicofísica. }\end{array}$ & $2 \mathrm{~B}$ \\
\hline
\end{tabular}

Fonte: Bezerra BR, et al., 2020. I Health 
A presente revisão integrativa sintetizou e avaliou os principais achados disponíveis na literatura sobre os efeitos da técnica de relaxamento muscular de Jacobson em indivíduos com sintomas de ansiedade. De modo geral, os estudos analisados demonstram em seus resultados que a técnica apresenta efeitos positivos, e que vem sendo realizada como método terapêutico em pessoas que apresentam sintomas de ansiedade em vários contextos.

Os resultados apontaram que o relaxamento muscular aplicado em pessoas com ansiedade, e outras comorbidades, obteve diminuição dessa sintomatologia, especialmente em indivíduos com várias doenças crônicas, como câncer (PIFARRÉ P, et al., 2015; PARÁSBRAVO P, et al., 2017; 2018; POURABOLI B, POODINEH Z, JAHANI Y, 2019), tensão psicofísica associada a processo de reabilitação psicomotora e neurológica (WILCZYŃSK D, et al., 2019; HAMPSON N, et al. 2019), obesidade submetidos à cirurgia bariátrica (FÉLIX MMS, et al., 2018), estresse ocupacional e ansiedade (BORGES EMN, FERREIRA TJR, 2013; FREITAS GR, et al., 2018; NORELLI SK, KREPPS JM, 2016; MERAKOU K, et al. (2019), pacientes cardíacos submetidos a cirurgia cardiovascular (GOMES MIS, CRUZ ICF, 2015), Endometriose (ZHAO L, et al. 2012)

A ansiedade é um problema emergente. Entre as consequências danosas para a vida dessas pessoas com sintomas ansiosos, cita-se o surgimento de emoções negativas que atuam mediando estímulos de estresse e padrões de reação emocional. Estes efeitos, todavia, podem ser minimizados por meio de métodos não farmacológicos, entre estes, encontram-se as técnicas de relaxamento muscular, que podem ser utilizadas para reduzir a ansiedade (WILCZYNSKA D, et al., 2019).

A técnica de relaxamento muscular, como evidenciado nos resultados deste estudo, é considerada um método terapêutico baseado em exercícios que proporcionam a redução de sintomas físicos e psicológicos de ansiedade, podendo ser realizada em ambientes domiciliares e de saúde, pelo próprio paciente, ou mediada por profissionais de saúde habilitados (NORELLI JK; KREEPS JM, 2019). Importa destacar que além da ansiedade, os participantes dos estudos apresentados nesta revisão apresentavam outros diagnósticos que contribuíam para o surgimento de sintomas ansiosos e de estresse.

Pessoas diagnosticadas e em tratamento para o câncer, por exemplo, apresentaram também dor, sintomas de isolamento, tristeza, baixa autoestima, distúrbios emocionais e alterações comportamentais. Em estudo realizado com estes pacientes utilizando técnicas de relaxamento muscular ao longo de quatro semanas, observou-se uma diminuição do consumo de drogas psicotrópicas e analgésicas ocasionada pelos benefícios psicológicos e físicos, melhoria na qualidade de vida e redução dos sintomas de ansiedade, estresse emocional e depressão com eficácia demonstrada até um mês após implementação da técnica (PARÁS-BRAVO P, et al., 2017; 2018; PIFARRÉ P, et al, 2015).

Outros estudos em pacientes com câncer demonstraram que o relaxamento muscular progressivo tem efeito significativo na diminuição da dor e fadiga (DIKMEN HÁ, TERZIOGLU F, 2019). Considerando que o nível de dor correlaciona-se negativamente com sintomas de ansiedade e com a qualidade de vida (CAPELA C et al., 2009), pode-se inferir que o uso da técnica de relaxamento diminui a sensação de dor e os sintomas de ansiedade, consequentemente diminuindo a necessidade do uso de medicação psicotrópica e analgésica, impactando positivamente na qualidade de vida de pessoas em tratamento de câncer.

Em contrapartida Pouraboli B, Poodineh Z, Jahani Y (2019) também colocam que o câncer pode causar estresse emocional e ansiedade nos pais que fazem acompanhamento no tratamento de filhos com câncer, causando sensação de fraqueza, redução da capacidade de atividade mental e física, além de insônia e incapacidade de desempenhar suas funções ocupacionais e sociais. Os resultados deste estudo predispõem que os cuidados de enfermagem centrados na família são de fundamental importância para apoiar os pais de crianças com câncer diante do estresse da doença. O desenvolvimento de programas para o treinamento de técnicas de relaxamento muscular melhorará o funcionamento da família e a saúde mental.

O câncer é considerado como uma doença crônica não transmissível, caracterizada como um problema contemporâneo de saúde pública mundial. Quando se apresenta de forma avançada, evolui com sinais e sintomas controláveis como dor, náuseas, vômitos, anorexia, fadiga, depressão, ansiedade, constipação, 
entre outros. Esses sintomas, principalmente o mental "ansiedade e depressão" tem acarretado intenso desconforto e grande impacto circunstancialmente impresumível para a qualidade de vida. Nesse sentido, os cuidados oferecidos ao paciente com câncer e sintomas psicológicos abalados, devem ser tratados de forma que vise uma melhor qualidade de vida, por meio da aplicação de intervenções, como a técnica de relaxamento muscular progressivo de Jacobson, com o escopo de mitigar o alivio da dor e dos sintomas psicológicos (FREIRE MEM, et al., 2018).

A realização do relaxamento muscular contribui para restaurar ou gerar sensação de bem-estar psicológico, físico, emocional e corporal no ser humano (NOVAIS PGN, 2015). Durante a técnica, a pessoa é favorecida movimenta diferentes grupos musculares, executando um processo psicofisiológico, no qual os sistemas psicológico e fisiológico interagem e, assim, relaxam (SERRA S, 2014).

O mecanismo de efeito do relaxamento consiste em desfazer a contração das fibras musculares, de modo que os indivíduos se tornam capazes de comandar diretamente os músculos. O bem-estar ocorre, então, devido à contração muscular, que estimula a liberação de monoaminas, e em sequência, acontece a distensão muscular, produzindo endorfinas, que geram sensação de prazer ao corpo humano (FREITAS GR, 2015).

A terapia de relaxamento muscular tem sido utilizada como método complementar ao tratamento medicamentoso em pessoas que vivem com tensão psicofísica e condições neurológicas a longo prazo, 0 que por sua vez pode levar a melhores resultados funcionais da neuro-reabilitação e diminuição dos sintomas ansiosos e depressivos. Wilczyńska D, Hampson N, et al. (2019) e Félix MMS, et al. (2018) afirmam que o relaxamento muscular ajuda a melhorar as condições funcionais de reabilitação, bem como comtempla as questões psicológicas consideradas como um abarreira no tratamento reabilitatório.

O relaxamento também garante a redução do estresse mental e os processos mentais associados por meio da supressão progressiva da tensão muscular, sendo um método não farmacológico que pode ser tão eficaz quanto um medicamento ansiolítico. Pois, a partir do momento em que se pode controlar diretamente os músculos e transitar de um estado de tensão para o de relaxamento em poucos minutos, o indivíduo passa a ter controle de suas tensões e ansiedade (PIFARRÉ P, et al., 2015).

Freitas P, et al. (2018) e Borges EMN, Ferreira TJR (2013) colocam que o estresse ocupacional pode causar inúmeros prejuízos para a saúde, como por exemplo, o surgimento de sintomas ansiosos, tensão, medo, angústia e aumento da pressão arterial. Estes autores utilizaram a técnica de relaxamento muscular, na oportunidade obtiveram respostas saudáveis frente a estímulos ambientais aversivos, favorecendo a melhoria da qualidade de vida.

Estudo internacional que buscou comparar dois tipos de intervenção, relaxamento muscular progressivo e Mindfulness, sobre a depressão, ansiedade e estresse, observou que os participantes de ambos os grupos apresentaram melhorias significativas para sintomas de ansiedade e estresse. Para a ansiedade, especificamente, os resultados apontaram melhora substancial entre aqueles do grupo que recebeu terapia de relaxamento muscular (CORBETT C; EGAN J; PILCH M, 2019).

De acordo com Paiva KCM, et al. (2019) os eventos estressores são desencadeados por meio de ações adotadas pelo indivíduo de modo a enfrentar os excessos percebidos, ou através de esforços cognitivos ou comportamentais. Assim, o apoio social é de fundamental importância, no sentido de reduzir esse estresse, pois compartilhar tempo com os amigos, parentes ou mesmo com outros profissionais já o ajuda bastante a ter um discernimento dos eventos vivenciados, e desta forma, o mesmo poderá buscar em manter o equilíbrio psíquico, como forma de suavizar os momentos de pressão.

Segundo Gomes MIS e Cruz ICF (2015) pacientes com problemas cardíacos e endometriose (Zhao L, et al. (2012) normalmente apresentam sintomas ansiosos em decorrência de sentimentos como incerteza, medo e angústia. Dentro desse contexto, a técnica de relaxamento muscular foi considerada como uma prescrição de enfermagem útil para redução desses sintomas, aplicada durante todo o período pré-operatório.

Nessa perspectiva, é válido destacar que o enfermeiro é um profissional primordial no tratamento de pessoas que necessitam de um cuidado mais centrado e holístico. Gomes MIS e Cruz ICF (2015) afirmam 
que os indicadores de ansiedade devem ser observados e identificados por meio das respostas comportamentais e psicológicas dos pacientes. Ressalta-se que profissionais de saúde devem se apropriar de técnicas interventivas para agravos em saúde mental apoiadas em boas evidências, com qualidade suficiente (BARBUI C, et al., 2020).

Portanto, os estudos analisados, apontaram que a utilização da Técnica de Relaxamento Muscular de Jacobson utilizada como método complementar ao tratamento medicamentoso as pessoas com sintomas de ansiedade que enfrentam um problema de saúde crônico foi bastante eficaz, garantindo, assim, redução dos sintomas de ansiedade e melhor qualidade de vida, pois o desenvolvimento de programas para o treinamento de técnicas de relaxamento muscular melhorará o funcionamento da saúde física e mental tanto do familiar quanto os indivíduos que vivem com doenças crônicas.

\section{CONSIDERAÇÕES FINAIS}

Diante da análise dos resultados desta revisão integrativa, conclui-se que a técnica de relaxamento muscular, enquanto terapia complementar, é um método eficaz e que atua de forma positiva na redução dos sintomas de ansiedade nos diversos contextos de saúde, bem como na melhoria da qualidade de vida e recuperação da saúde do usuário assistido por profissionais da saúde, em especial pelo enfermeiro, que exerce papel crucial na promoção de métodos e técnicas que atuam na redução da ansiedade.

\section{REFERÊNCIAS}

1. BARBIANI R, NORA CRD, SCHAFER R. Práticas do enfermeiro no contexto da atenção básica: scoping review. Revista Latino-Americana de Enfermagem, 2016; 16(24): 2721-2733.

2. BARBUI C, et al. Efficacy of psychosocial interventions for mental health outcomes in low-income and middle-income countries: an umbrella review. Lancet Psychiatry, 2020; 7(2): 162-172.

3. BORGES EMN, FERREIRA TJR. Relaxamento: Estratégia de intervenção no stress. Revista Portuguesa de Enfermagem de Saúde Mental, 2013: 10: 37-48.

4. BRASIL. Portaria no 3.088, de 23 de dezembro de 2011. Institui a Rede de Atenção Psicossocial para pessoas com sofrimento ou transtorno mental e com necessidades decorrentes do uso de crack, álcool e outras drogas, no âmbito do Sistema Único de saúde (SUS). Brasília; 2011.

5. CAIXETA PPP. Ansiedade na atenção primária, como lidar? MG. Trabalho de Conclusão de Curso (Especialização em Estratégia de Saúde da Família) - Universidade Federal do Triângulo Mineiro, Uberaba, 2014; 26 p.

6. CAPELA C, et al. Associação da qualidade de vida com dor, ansiedade e depressão. Fisioter. Pesqui. [online]. 2009; 16 (3):263-268.

7. CENTRE FOR EVIDENCE-BASED MEDICINE. Levels of evidence, Oxford, 2009. Disponível em: http://www.cebm. net/oxford-centre-evidence-based-medicine-levels-evidence-march-2009/. Acesso em: 20 nov. 2019.

8. CORBETT C, EGAN J, PILCH M. A randomised comparison of two 'Stress Control' programmes: Progressive Muscle Relaxation versus Mindfulness Body Scan. Mental Health \& Prevention, 2019; 15: 200163.

9. DIKMEN HA, TERZIOGLU F. Effects of Reflexology and Progressive Muscle Relaxation on Pain, Fatigue, and Quality of Life during Chemotherapy in Gynecologic Cancer Patients. Pain Manag Nurs. 2019; 20(1):47-53.

10. FÉLIX MMS, et al. Terapia de relaxamento muscular com imagem guiada sobre ansiedade pré-operatória: ensaio clínico randomizado. Rev. Latino-Americana de Enfermagem, 2018; 26: 1-10.

11. FREITAS GR, CALAIS SL, CARDOSO HF. Estresse, ansiedade e qualidade de vida em professores: efeitos do relaxamento progressivo. Psicologia Escolar e Educacional, 2018; 22(2): 319-326.

12. FREIRE, MEM et al. Qualidade de vida relacionada à saúde de pacientes com câncer em cuidados paliativos. Texto contexto - enferm. 2018; 27(2): e5420016.

13. FREITAS GR. Estresse, ansiedade e qualidade de vida em professores: efeitos do relaxamento progressivo. Dissertação (Mestrado em Psicologia do Desenvolvimento e Aprendizagem) - Universidade Estadual Paulista Júlio de Mesquita Filho, Bauru, 2015; 49p.

14. GOMES MIS, CRUZ ICF. Relaxamento muscular progressivo: prescrição de enfermagem para o diagnóstico de ansiedade pré-operatória. Boletim Nepae-Nesen, 2015; 12(2): 1-11.

15. KESTENBERG, C. C. F. et al. Estresse em graduando de enfermagem: técnicas de relaxamento para lidar com fatores estressores. Interagir: pensando a extensão, n. 17-19, p. 37-43, 2014. 
16. MOREIRA MIB, CAMPOS TO. Ações de saúde mental na rede de atenção psicossocial pela perspectiva dos usuários. Saúde e Sociedade, 2017; 26(2): 462-474.

17. MOHER D. et al. Preferred reporting items for systematic reviews and meta-analyses: the PRISMA statement. PLoS Med. 2009 21;6(7):e1000097.

18. MULLER JL, et al. Transtorno de ansiedade social: um estudo de caso. Contextos Clínicos, 2015; 8(1): 67-78.

19. NORELLI SK, KREPPS JM. Técnicas de relaxamento. StatPearls. Disponível em: https://www.ncbi.nlm.nih.gov/books/NBK513238/. Acesso em: 30 de out. 2019.

20. NOVAIS PGN. Efeito do relaxamento muscular progressivo como intervenção de enfermagem na qualidade do sono, depressão e estresse em pessoas com esclerose múltipla. Dissertação (Mestrado em Enfermagem) - Universidade Federal do Espírito Santo, Vitória, 2015; 147p.

21. PAIVA KCM, et al. Estresse Ocupacional e Síndrome de Burnout: Estudo em uma Indústria do Estado de Minas Gerais. Revista FSA, 2019; 16(2): 272-295.

22. PARÁS-BRAVO $P$, et al. O impacto das técnicas de relaxamento muscular na qualidade de vida de pacientes com câncer, medido pelo questionário. FACT-G. PLoS One, 2017; 12(10).

23. PARÁS-BRAVO $P$, et al. Does Jacobson's relaxation technique reduce consumption of psychotropic and analgesic drugs in cancer patients? A multicenter pre-post intervention study. BMC Complementary and Alternative Medicine, 2018; 18(1): 2-9.

24. PIFARRÉ P, et al. Diazepam and Jacobson's progressive relaxation show similar attenuating short-term effects on stress-related brain glucose consumption. European Psychiatry, 2015; 30(2): 187-192.

25. SERRA S. Intervenções de Enfermagem na Pessoa com sintomatologia depressiva: o uso do relaxamento. Dissertação (Mestrado em Enfermagem, Saúde Mental e Psiquiatria) - Instituto Politécnico de Setúbal. Portugal, 2014; $132 \mathrm{p}$.

26. SOUZA MT, SILVA MD, CARVALHO R. Integrative review: what is it? How to do it? Einstein, 2010; 8(1): 102-6.

27. VASCONCELOS LHS. Avaliação da eficácia da técnica de relaxamento muscular progressivo de Jacobson na redução de fadiga em uma trabalhadora de enfermagem, SP. Dissertação (Mestrado em Gerenciamento em Enfermagem) Escola de Enfermagem. Universidade de São Paulo, São Paulo, 2014; 107 p.

28. WHO - World Health Organization. Depression and Other Common Mental Disorders: Global Health Estimates. Geneva: World Health Organization, 2017.

29. WILCZYŃSKA D, et al. Evaluation of the effectiveness of relaxation in lowering. The level of anxiety in young adults a pilot study. International Journal of Occupational Medicine and Environmental Health, 2019; 32(6): 817-824. 